PITT-93-4

\title{
DYNAMICS OF PHASE TRANSITIONS INDUCED BY A HEAT BATH
}

\author{
Da-Shin Lee and Daniel Boyanovsky \\ Department of Physics and Astronomy \\ University of Pittsburgh \\ Pittsburgh, P. A. 15260, U.S.A.
}

\begin{abstract}
We study the non-equilibrium dynamics of a symmetry restoring phase transition in a scalar field theory, the "system", linearly coupled to another scalar field taken as a "heat bath". The "system" is initially in an ordered low temperature phase, and the heat bath is at a temperature close to the critical temperature for the system. We estimate the time at which the phase transition to the disordered (symmetric) phase takes place. We derive, and integrate the one-loop effective equations of motion for the order parameter that include the effects of the heat bath. A semiclassical Langevin equation is derived and it is found that it contains a non-dissipative, non-Markovian kernel, the noise term is colored and correlated on time scales determined by the temperature of the heat bath. The range of validity of the Langevin equation and a consistent procedure to incorporate corrections are discussed.
\end{abstract}

\section{Introduction and Motivation}

Non-equilibrium aspects of quantum field theory and statistical mechanics are beginning to receive considerable attention, for example within the context of structure formation in inflationary cosmology [1, 2, 3, 4], dissipation and decoherence in quantum cosmology [5, 6], in the theory of quantum brownian motion [7, 8, 9], and field theory in noisy environments [10] to cite but a few.

An important setting in which statistical mechanics out of equilibrium will play a very important role is in the description of the dynamics of phase transitions in the early universe. Recently the effective evolution equations to one loop order for the order parameter 11] and the process of domain formation and growth[12] during a typical second order phase transition had been studied. 
In this article we study the non-equilibrium aspects of a symmetry restoring phase transition induced by coupling a system originally at low temperature in a broken symmetry state to a heat bath at a temperature close to the critical temperature of the system. Furthermore we derive a Langevin equation for the system that incorporates the effects of the heat bath, determine its range of validity, and provide a consistent procedure to incorporate corrections to the Langevin description.

Phenomenological Langevin equations had been recently used in a variety of field theoretical applications: for a semiclassical non-equilibrium analysis of thermal activation [13, 14], and the dynamics of kink-pair production in a heat bath 15. These phenomenological Langevin equations incorporate a linear friction term and an uncorrelated (white) noise that satisfy the classical limit of the fluctuation-dissipation theorem.

Our motivations for this article are twofold. First we want to study the non-equilibrium dynamics of a phase transition induced by coupling a system to a heat bath from a firstprinciple calculation. Secondly we want to qualify and quantify the situations in which a phenomenological Langevin equation with friction and white noise is justified, again, by establishing a first-principle derivation of the field-theoretical Langevin equation, its range of validity and possible corrections. One of the physically relevant questions that we want to address is about the time scales in which a "system" equilibrates with a heat-bath.

An equilibrium description of a thermodynamic system assumes that the degrees of freedom under consideration had reached thermal equilibrium with a "heat bath" or reservoir, and that the system is observed at times much larger than its typical relaxation time. When all the dynamical transient effects have had enough time to relax, the final state of the system would be that of equilibrium and the final state will not be sensitive to details of initial conditions or correlations, but only to the global thermodynamic properties of the "heat bath", like temperature etc.

Although these assumptions are commonly used and clearly correct in a wide variety of experimental situations, their extrapolation to the realm of a Quantum Field Theory in extreme environments like the Early Universe or Heavy Ion Collisions is not only unclear but perhaps unwarranted a-priori. In particular, the concept of a "heat bath" is somewhat obscure when studying a field theory as a "system". Strictly speaking, a "heat bath" or reservoir, must have certain characteristics that allow to separate in a well defined manner the reservoir from the system, in particular the characteristic relaxation times of the heat bath must be much shorter than those of the system, secondly, the reservoir must have an infinite specific heat (a condition necessary for the equivalence between a microcanonical and canonical description) and thus infinitely many degrees of freedom. 
The second condition is fulfilled if the heat bath is a quantum field theory, but if the "system" under consideration is also a quantum field theory, both system and bath have infinitely many degrees of freedom and the separation becomes somewhat artificial.

The separation of time scales presents another conceptual difficulty in field theory because both system and bath have degrees of freedom at all energy scales with typically comparable density of states at large energies (determined solely by the relativistic dispersion relation).

If the relaxation time for the heat bath is much shorter than that of the system, one may ignore the dynamics of the heat-bath and only ask questions about the system. Effectively this is achieved by "tracing over" the degrees of freedom of the bath.

In this article we examine the situation in which a self interacting scalar field is coupled to another scalar field at a particular time. Prior to this time both fields are uncoupled and assumed to be described by a thermal equilibrium ensemble, but at different temperatures. This initial condition is somewhat arbitrary and unjustified, but our purpose is to study the time evolution of one of the fields (the self-interacting scalar field) taken to be the "system", and trace out the other field, taken to be the "heat bath". If the "system" field is at zero temperature in a state of broken symmetry, how long does it take to produce the phase transition and to restore the symmetry if the "heat bath" is at a temperature close to the critical??.

A very succesful approach to study a Brownian particle in a heat bath or environment is provided by the Langevin equation. The effect of the heat bath is to introduce a friction term and a stochastic noise (typically assumed to be uncorrelated) both being related by the fluctuation-dissipation theorem.

We want to study if such a description is suitable for a field theory and if so under which circumstances, establishing the range of validity of such description and possible corrections.

In section II the essential elements to study field theory out of equilibrium are introduced. In section III we study the dynamics of the symmetry restoration phase transition induced by the heat bath. Section IV presents the derivation of the Langevin equation, discusses the range of validity and the formal procedure to incorporate corrections. Our conclusions are summarized in section $\mathrm{V}$. Two appendices present many technical details.

\section{Evolution out of Equilibrium: Real Time Analysis}

As mentioned in the introduction, our aim is to study the time evolution of the "system" when it is coupled to a "heat bath" at a different temperature. By "system" we now refer to the simplified case of a self-interacting scalar field, and by "bath" we will take for simplicity 
the case of a free massive field with a mass much larger than the mass of the quanta of the "system", so as to justify that the time scales of the heat bath are much shorter than those of the system. Eventually we will take the temperature of the heat bath to be much larger than the masses (of both system and bath). In this situation, we can relax the constraint that the mass of the particles in the heat bath be much larger than that of the system.

One can avoid having to face the question of how the "bath" acquired its temperature, by assuming that the bath degrees of freedom are in a highly excited state corresponding to a microcanonical ensemble with an energy density $\mathcal{E} \gg m_{\sigma}$ with $m_{\sigma}$ the mass of the particles in the bath. Under these circumstances one can pass from the microcanonical ensemble at this energy density to a canonical ensemble by a Laplace transform. The temperature of the equivalent canonical ensemble is $T_{\sigma} \approx \mathcal{E}^{\frac{1}{4}}$. We will use the description in terms of the canonical ensemble.

We will also require that the coupling of the system to the bath satisfies the following conditions: i) locality ii) manifest Lorentz covariance, iii) maintains the renormalizability of the scalar field theory.

For calculational simplicity we will also assume that the coupling is linear in both fields. The simplification of a "free field bath" and linear coupling will allow us to present detailed calculations, these restrictions may be relaxed at the expense of further complications.

Thus our situation may be modelled by introducing the time dependent Hamiltonian

$$
H(t)=H_{\Phi}+H_{\sigma}+H_{I}(t)
$$

where

$$
H_{\Phi}=\int_{V} d^{3} x\left\{\frac{1}{2} \Pi_{\Phi}^{2}(x)+\frac{1}{2}(\vec{\nabla} \Phi(x))^{2}+\frac{1}{2}\left(-\mu^{2}\right) \Phi^{2}(x)+\frac{\lambda}{4 !} \Phi^{4}(x)\right\}
$$

is the Hamiltonian of the self-interacting field that will be taken as the "system", and $\mu^{2}$ is chosen to be positive with the purpose of studying non-equilibrium aspects of phase transitions.

$$
H_{\sigma}=\int_{V} d^{3} x\left\{\frac{1}{2} \Pi_{\sigma}^{2}(x)+\frac{1}{2}(\vec{\nabla} \sigma(x))^{2}+\frac{1}{2} m_{\sigma}^{2} \sigma^{2}(x)\right\}
$$

is the Hamiltonian for the free field taken to be as the "bath". Finally $H_{I}(t)$ is the interaction Hamiltonian given by

$$
H_{I}(t)=g \Theta(t) \int_{V} d^{3} x \Phi(x) \sigma(x)
$$


To completely determine the time evolution of the "system- bath", we need to specify the initial conditions. We assume (without any a-priori justification), that for $t<0$ both the system and bath are in thermal equilibrium at temperatures $T_{\Phi} ; T_{\sigma}$ respectively. Furthermore we will assume that the field $\Phi$ is in a broken symmetry state at $T_{\Phi} \approx 0$ and that $T_{\sigma} \gg m_{\sigma}^{2}, \mu^{2}$. Notice that the coupling constant $g$ has dimensions of $(\text { mass })^{2}$, and a perturbative expansion in this coupling, will involve the ratio of this coupling to a particular energy scale of the heat bath. Under the assumption that the temperature of the bath is much larger than the masses of the bath and system field, we expect the perturbative expansion parameter to actually be in terms of the dimensionless ratio $\tilde{g}=g / T_{\sigma}^{2}$. Furthermore, in this limit, we expect the characteristic time (relaxation time) of the heat-bath to be $t_{\text {bath }} \approx 1 / T_{\sigma}$.

For the study of the dynamics in real time, the quantity of interest is the density matrix in the Schroedinger picture. For $t \leq 0$ the system and bath are uncoupled and the initial density matrix is given by

$$
\hat{\rho}(0)=e^{-\beta_{\Phi} H_{\Phi}} \otimes e^{-\beta_{\sigma} H_{\sigma}}
$$

where $\beta=1 / K_{B} T$ for the respective temperatures. In the Schroedinger picture, the density operator evolves in time as

$$
\hat{\rho}(t)=U(t) \hat{\rho}(0) U^{-1}(t)
$$

with $U(t)$ the time evolution operator.

As mentioned previously, our aim is to understand the dynamics of a field interacting with a heat bath. Physically we are interested in correlation functions of the scalar field $\Phi$, but not on any properties of the heat bath. Thus, we proceed to trace over the degrees of freedom of the heat bath, obtaining an effective theory for the scalar field.

This procedure, starting from a closed "system-heat bath" and tracing over the reservoir degrees of freedom leads to the description of the "system" as an open environment, and is fundamentally equivalent to passing from a microcanonical to a canonical description.

The procedure of tracing out (performing the path integral) the bath degrees of freedom yields to a "reduced density matrix" for the system. Although the full density matrix satisfies the Liouville equation with the full Hamiltonian, the reduced density matrix satisfies a Fokker-Planck equation[16, 17].

This procedure originally proposed by Feynman and Vernon 18], has been used repeateadly in the literature [16], in particular in the study of dissipative effects in quantum mechanics and for the consistent treatment of quantum Brownian motion[19]. There are 
some major differences between our work and past work on quantum brownian motion and dissipative systems. In the usual treatment of quantum brownian motion[16], a particular density of states must be assumed for the heat bath. The choice of density of states leads to different dissipative behavior, and is reflected in the correlation functions of the heat bath through the fluctuation-dissipation relation.

In our case, the nature of the heat-bath, and its coupling to the system, are constrained by the requirements of locality, relativistic covariance, and renormalizability, which severely constrain the density of states of the heat bath and the possible couplings to the system.

The expectation value of an arbitrary operator $\langle\mathcal{O}\rangle(t)$ is given by

$$
\langle\mathcal{O}\rangle(t)=\frac{\operatorname{Tr} \hat{\rho}(t) \mathcal{O}}{\operatorname{Tr} \hat{\rho}(0)}
$$

It is more illuminating and convenient for our purposes to cast the above expression in terms of the time evolution operator $U$. This is achieved by first choosing a large negative time $T<0$ (not to be confused with temperature), for which $U(T)=U_{\Phi}(T) U_{\sigma}(T)$ where $U_{i}(T)=\exp \left[-i H_{i} T\right]($ for $i=\Phi ; \sigma)$ we may write $\exp \left[-\beta_{i} H_{i}\right]=\exp \left[-i H_{i}\left(T-i \beta_{i}-T\right)\right]=$ $U_{i}\left(T-i \beta_{i}, T\right)$.

Inserting in the trace $U^{-1}(T) U(T)=1$, commuting $U^{-1}(T)$ with $\hat{\rho}(0)$ and using the composition property of the evolution operator, the expectation value (7) becomes

$$
\langle\mathcal{O}\rangle(t)=\frac{\operatorname{Tr}\left\{U_{\Phi}\left(T-i \beta_{\Phi}, T\right) U_{\sigma}\left(T-i \beta_{\sigma}, T\right) U^{-1}(T, t) \mathcal{O} U(t, T)\right\}}{\operatorname{Tr}\left\{U_{\Phi}\left(T-i \beta_{\Phi}, T\right) U_{\sigma}\left(T-i \beta_{\sigma}, T\right)\right\}}
$$

It proves convenient to choose a large positive time $T^{\prime}$ and to insert $U\left(t, T^{\prime}\right) U\left(T^{\prime}, t\right)=1$ (with $\mathrm{U}$ the full time evolution operator) to the left of $\mathcal{O}$ in (8) to extend the contour in the numerator to an arbitrary large positive time $T^{\prime}$ [20]. Finally, the thermal expectation value of the operator $\mathcal{O}$ is

$$
\langle\mathcal{O}\rangle(t)=\frac{\operatorname{Tr}\left\{\left[U_{\Phi}\left(T-i \beta_{\Phi}, T\right) U_{\sigma}\left(T-i \beta_{\sigma}, T\right)\right] U\left(T, T^{\prime}\right) U\left(T^{\prime}, t\right) \mathcal{O} U(t, T)\right\}}{\operatorname{Tr}\left\{U_{\Phi}\left(T-i \beta_{\Phi}, T\right) U_{\sigma}\left(T-i \beta_{\sigma}, T\right)\right\}}
$$

In the complex time plane, the numerator represents the following process: from $T<0$, evolve in time up to $t$, insert the operator $\mathcal{O}$, evolve further up to $T^{\prime}$, and backwards from $T^{\prime}$ to $T$, finally down the negative imaginary axis to $T-i \beta_{\Phi}$ for $\Phi$ field $\left(T-i \beta_{\sigma}\right.$ for the $\sigma$ field) depicted in figure (1). The denominator just evolves along the negative axis from $T$ to $T-i \beta_{\Phi ; \sigma}$ for $\Phi ; \sigma$. Eventually we will take the arbitrary times $T \rightarrow-\infty ; T^{\prime} \rightarrow \infty$.

The insertion of the operator $\mathcal{O}$ may be obtained as usual by introducing external sources coupled to the particular operator. 
Since we propose to use perturbation theory to study the non-equilibrium correlation functions, it proves more convenient to introduce different source terms for all the time evolution operators in the trace. The sources will be different on the different branches along the complex time contour. However, we do not introduce source terms for the field $\sigma$ as it will be traced out, and no correlation functions of this field will be computed. We are thus led to consider the following generating functional

$$
Z\left[J_{\Phi}^{+}, J_{\Phi}^{-}, J_{\Phi}^{\beta}\right]=\operatorname{Tr}\left\{\left[U_{\Phi}\left(T-i \beta_{\Phi}, T ; J_{\Phi}^{\beta}\right) U_{\sigma}\left(T-i \beta_{\sigma}, T\right)\right] U\left(T, T^{\prime} ; J_{\Phi}^{-}\right) U\left(T^{\prime}, T ; J_{\Phi}^{+}\right)\right\}
$$

where $J_{\Phi}^{ \pm}$are the source terms for the field $\Phi$ along the forward $(+)$ and backward $(-)$ segments of the contour, and the denominator in (8) is given by $Z\left[0,0, J^{\beta}\right]$. This formalism allows to study more general situations with different choices of $\rho(0)$.

The necessity for these generating functionals to study non-equilibrium quantum statistical mechanics was originally proposed by Keldysh 22] and Schwinger 21] and has been used in the literature very

often 23, 24, 25, 26, 27, 28, 29]. The trace over the $\sigma$ field (functional integral) may be easily performed because it is a free field linearly coupled to the scalar field $\Phi$, which acts as a "source term" for the $\sigma$ field, yielding to a non-local quadratic influence functional $18 \rrbracket$.

$$
\mathcal{F}\left[\Phi^{+}, \Phi^{-}\right]=\exp \left\{-\frac{i}{2} g^{2} \int_{0}^{T^{\prime}} d t_{1} \int_{0}^{T^{\prime}} d t_{2} \Phi^{a}\left(t_{1}\right) D_{\sigma}^{a b}\left(t_{1}, t_{2}\right) \Phi^{a}\left(t_{2}\right)\right\}
$$

with a sum over the indices $a, b=+,-$, corresponding to the forward $T \rightarrow T^{\prime}$ and backward $T^{\prime} \rightarrow T$ branches of the contour respectively, and $\left(D_{\sigma}^{a b}\left(t_{1}, t_{2}\right)\right)$ are the Green's functions on the contour for the "heat-bath" given in appendix A.

We relegate most of the technical details to the appendices, where we show that the final form of the generating functional becomes

$$
\begin{aligned}
Z\left[J_{\Phi}^{+}, J_{\Phi}^{-}\right]= & \exp \left\{i \frac{\lambda}{4 !} \int_{-\infty}^{\infty} d t\left[\left(-i \frac{\delta}{\delta J_{\Phi}^{+}}\right)^{4}-\left(i \frac{\delta}{\delta J_{\Phi}^{-}}\right)^{4}\right]\right\} \mathcal{F}\left[-i \frac{\delta}{\delta J_{\Phi}^{+}}, i \frac{\delta}{\delta J_{\Phi}^{-}}\right] \times \\
& \exp \left\{-\frac{i}{2} \int_{-\infty}^{\infty} d t_{1} \int_{-\infty}^{\infty} d t_{2} J_{\Phi}^{a}\left(t_{1}\right) D_{\Phi}^{a b}\left(t_{1}, t_{2}\right) J_{\Phi}^{a}\left(t_{2}\right)\right\}
\end{aligned}
$$

with $a, b=+,-$.

The Green's functions on the contour and their properties are analyzed in appendix A. We are now in condition to study the dynamics in real time for the field $\Phi$ in the presence of the "heat-bath". As mentioned in the introduction, one of our principal motivations is to 
address the fundamental question of symmetry restoration by the heat bath. In particular, if the system is originally at very low temperatures (or zero temperature) in a broken symmetry state and is suddenly coupled (at $t=0$ ) to a "heat bath" in equilibrium at a temperature close to the critical temperature, how long does it take for the symmetry to be restored?? What are the relevant time scales for the dynamics of symmetry restoration?? The answer to the last question is certainly far from obvious. There are several widely different time scales in the problem, the typical time of the heat bath (determined by the mass and temperature of the $\sigma$ field), the mass and temperature of the scalar field (system), and finally the coupling system-bath. It is not a-priori obvious which time scale or combination thereof will determine the dynamics of the process of symmetry restoration. However, on physical grounds, we expect that for very weak coupling between the system and the heat bath, this time will be very large. This intuitive argument must be qualified however. The dimensionless coupling is expected to be $g / T_{\sigma}^{2}$, and the transition time is expected to be "large" on time scales of the heat bath. Thus when the temperature of the bath is much larger than the masses of both system and bath we expect the "transition time" $t_{c}$ (the time at which the symmetry restoration phase transition occurs) to be such that

$$
t_{c} T_{\sigma} \gg 1
$$

\section{Symmetry Restoration}

Usually the physics of a phase transition is studied by means of the static effective potential (free energy density), as the position of the minima of the effective potential determine the thermodynamic equilibrium states of the system. If the minima correspond to a nonzero value of the expectation value (or thermal average) of the scalar field the symmetry is spontaneously broken, otherwise the symmetry is restored. The vacuum expectation value or thermal ensemble of the scalar field serves as the order parameter for the transition. In a translational invariant theory this order parameter is independent of the spatial coordinates, and in equilibrium it must be independent of time.

In our case, we couple suddenly the system to the heat-bath at time $t=0$ and let the system evolve in time with the full interaction Hamiltonian. Clearly this is not an equilibrium situation, as the initial density matrix does not commute with the full Hamiltonian for $t>0$. The static effective potential is not the proper quantity to study the dynamics of the phase transition and one should invoke the effective action to account for the real time dependence 
of the situation. Because translational invariance is still preserved, the order parameter

$$
\langle\Phi(\vec{x}, t)\rangle=\frac{\operatorname{Tr} \hat{\rho}(t) \Phi(\vec{x}, t)}{\operatorname{Tr} \hat{\rho}(0)}=\phi(t)
$$

only depends on time (and certainly temperature).

Initially the scalar field is in equilibrium in the broken symmetry phase at very low temperature $\left(T_{\Phi} \ll T_{c}=\sqrt{24 \mu^{2} / \lambda}\right)$ and is suddenly coupled to the heat bath whose temperature is $T_{\sigma} \approx T_{c} \gg m_{\sigma}, \mu$. The order parameter will evolve in time and the phase transition will occur when the order parameter becomes zero or begins to oscillate around zero.

Obtaining the effective action even to one-loop order is clearly an imposing task, the same information about the phase transitions is obtained from the effective evolution equations for the order parameter. These equations of motion are formally obtained as a variational principle from the effective action, by requiring that the functional derivative of the effective action with respect to the order parameter vanishes. It turns out that it is much easier to obtain the effective equations of motion for the order parameter from the non-equilibrium formalism. This is achieved by using the tadpole method[30] and applied to this non-equilibrium situation by shifting the fields $\Phi^{ \pm}$on the forward and backward branches as follows [11, 12] (see appendix B)

$$
\Phi^{ \pm}(\vec{r}, t)=\phi(t)+\varphi^{ \pm}(\vec{r}, t)
$$

The reason for shifting both $( \pm)$ fields by the same configuration, is that $\phi$ enters in the time evolution operator as a background c-number field, and evolution forward and backwards are now considered in this background. The effective equations of motion are obtained by requiring that

$$
\left\langle\varphi^{ \pm}(\vec{r}, t)\right\rangle=0
$$

These two conditions consistently result in the same evolution equation for the order parameter $\phi$ as a consequence of the cyclic property of the trace.

As explained in appendix B the evolution equations are obtained by expanding the action in (51) around $\phi(t)$, the quadratic terms for $\varphi^{ \pm}$will define the propagators and the higher order terms will be treated in perturbation theory. To one loop order we find

$$
\begin{aligned}
\frac{d^{2}}{d t^{2}} \phi(t)+\left(-\mu^{2}\right) \phi(t)+\frac{\lambda}{3 !} \phi^{3}(t) & -\frac{g^{2}}{m_{\sigma}} \int_{0}^{t} d t^{\prime} \sin \left[m_{\sigma}\left(t-t^{\prime}\right)\right] \phi\left(t^{\prime}\right) \\
& +\frac{\lambda}{2} \phi(t) \int \frac{d^{3} k}{(2 \pi)^{3}}\left\langle\varphi_{\vec{k}}^{+}(t) \varphi_{-\vec{k}}^{+}(t)\right\rangle=0
\end{aligned}
$$


The retarded kernel in the above expression is arising from the influence functional, notice that it does not depend on the temperature of the heat bath, this is a consequence of the fluctuation- dissipation theorem. This kernel is non-Markovian (has memory), but perhaps more importantly it is non-dissipative.

We will see later that this is the retarded kernel entering in the Langevin equation that describes the effective semiclassical evolution equations in the presence of the heat bath.

The one-loop term $\left\langle\varphi_{\vec{k}}^{+}(t) \varphi_{-\vec{k}}^{+}(t)\right\rangle$ is recognized as the spatial Fourier transform of the equal time two-point correlation function of the fluctuations. This correlation function is obtained by inverting the operator of quadratic fluctuations.

The quadratic terms that define these propagators, however, depend on the time dependent order parameter $\phi(t)$, and also receive a contribution from the influence functional (that mixes $\varphi^{ \pm}$). The identification of the "free propagator" is extremely difficult as one must find the inverse of the time dependent quadratic operator for the fluctuations. This involves summing the Dyson series for the mixing term in the influence functional. Because of the complicated time dependence we were unable to sum up the Dyson series and have to content ourselves with a perturbative expansion for both the time dependence of the order parameter and the contribution of the influence functional. Thus we write

$$
\begin{aligned}
-\mu^{2}+\frac{\lambda}{2} \phi^{2}(t) & =M^{2}+\frac{\lambda}{2}\left(\phi^{2}(t)-\phi^{2}(0)\right) \\
M^{2} & =-\mu^{2}+\frac{\lambda}{2} \phi^{2}(0)
\end{aligned}
$$

with $\phi^{2}(0)=6 \mu^{2} / \lambda$ being the minimum of the tree level potential. We identify the first term on the right hand side of (17) as the mass of quanta in the broken symmetry state. The second term will necessarily be of order $\lambda g^{2}$ or higher, thus $\mathcal{O}(\lambda)$ smaller than the term arising from the influence functional because the time dependence is induced by the coupling to the heat bath. As may be seen from (16), such a term will contribute to $\mathcal{O}\left(\lambda^{2} g^{2}\right)$ to the evolution equation. We will consider only the first order correction in $g^{2}$ to the propagator arising from the influence functional, a typical diagram is depicted in figure 2(b). The one loop contributions to this order to (16) are depicted in Figure 2(c). Thus we will consistently neglect to this order the second term on the right hand side of (17) (time dependence of the order parameter).

The first one-loop contribution depicted in Figure 2(c) is the familiar term, it is time independent and gives the usual result in terms of the temperature of the field $\Phi$, for our purposes assumed to be $T_{\Phi} \approx 0$. We will absorb this term in a renormalization of the bare parameters, $\mu^{2} ; \lambda$. 
Then we define consistently to this order the time dependent mass

$$
\begin{aligned}
m^{2}(t) & =\left(-\mu_{R}^{2}\right)+\Delta m(t) \\
\Delta m(t) & =\frac{\lambda_{R}}{2} \int \frac{d^{3} k}{(2 \pi)^{3}}\left\langle\varphi_{\vec{k}}^{+}(t) \varphi_{-\vec{k}}^{+}(t)\right\rangle_{g^{2}}
\end{aligned}
$$

where the $\mathcal{O}\left(g^{2}\right)$ contribution to the one-loop correlation function is depicted in figure 2(b). A simple analysis reveals, that for $t \neq 0$, this one loop diagram is ultraviolet finite, this contribution vanishes at $t=0$. Thus there are no further renormalizations (up to this order) induced by the coupling to the heat bath.

In the limit of $T_{\Phi} \approx 0$ we find

$$
\Delta m(t)=\Delta m^{(0)}(t)+\Delta m^{\left(T_{\sigma}\right)}(t)
$$

where

$$
\begin{aligned}
\Delta m^{(0)}(t) & =\frac{\lambda}{2} g^{2} \int \frac{d^{3} k}{(2 \pi)^{3}} \frac{1}{\omega_{k}^{\prime 2} \tilde{\omega}_{k}} \int_{0}^{t} d t_{1} \int_{0}^{t_{1}} d t_{2} \sin \left[\omega_{k}^{\prime}\left(t-t_{1}\right)\right]\left[\sin \left[\tilde{\omega}_{k}\left(t_{1}-t_{2}\right)\right] \cos \left[\omega_{k}^{\prime}\left(t-t_{2}\right)\right]\right. \\
& \left.+\cos \left[\tilde{\omega}_{k}\left(t_{1}-t_{2}\right)\right] \sin \left[\omega_{k}^{\prime}\left(t-t_{2}\right)\right]\right] \\
m_{\Delta}^{(T \sigma)}(t) & =\lambda g^{2} \int \frac{d^{3} k}{(2 \pi)^{3}} \frac{n_{\sigma}\left(\tilde{\omega}_{k}\right)}{\omega_{k}^{\prime 2} \tilde{\omega}_{k}} \int_{0}^{t} d t_{1} \int_{0}^{t_{1}} d t_{2}\left\{\sin \left[\omega_{k}^{\prime}\left(t-t_{1}\right)\right] \cos \left[\tilde{\omega}_{k}\left(t_{1}-t_{2}\right)\right]\right. \\
& \left.\times \sin \left[\omega_{k}^{\prime}\left(t-t_{2}\right)\right]\right\} \\
\omega_{k}^{\prime} & =\sqrt{\vec{k}^{2}+M^{2}} ; \tilde{\omega}_{k}=\sqrt{\vec{k}^{2}+m_{\sigma}^{2}}
\end{aligned}
$$

Let us first consider the finite temperature contribution. For this purpose it proves convenient to introduce the following dimensionless variables,

$$
x=\frac{k}{T_{\sigma}} \quad, \quad \tau=T_{\sigma} t
$$

After performing the time integrals in the high temperature limit $\left(T_{\sigma} \gg m_{\sigma} ; M^{2}\right)$, the finite temperature contribution (22) becomes

$$
\Delta m^{\left(T_{\sigma}\right)}(\tau)=\frac{\lambda T_{\sigma}^{2}}{2 \pi^{2}}\left(\frac{g^{2}}{T_{\sigma}^{4}}\right) \int_{0}^{\infty} d x \frac{1}{x\left(e^{x}-1\right)}\left\{\frac{1}{16 x^{2}}[1-\cos (2 x \tau)]+\frac{\tau^{2}}{8}\left[1-\frac{\sin (2 x \tau)}{x \tau}\right]\right\}
$$

This expression clearly shows that in this limit, the time scale for the heat bath is $1 / T_{\sigma}$. As argued previously, we expect that for small system-bath coupling $\left(g / T_{\sigma}^{2} \ll 1\right)$ the transition time $t_{c} \gg 1 / T_{\sigma}$. 
For $\tau \gg 1$, the integrand in (25) is sharply peaked at $x \approx 0$ allowing a saddle-point approximation to the integral. We find for $\tau \gg 1$

$$
\Delta m^{\left(T_{\sigma}\right)}(\tau) \approx 0.012 \tau^{3} \lambda T_{\sigma}^{2}\left\{\frac{g^{2}}{T_{\sigma}^{4}}\right\}
$$

In the same limit we estimate the contribution coming from the zero-temperature part to be

$$
\Delta m^{(0)}(\tau) \approx 0.013 \tau^{2} \lambda T_{\sigma}^{2}\left\{\frac{g^{2}}{T_{\sigma}^{4}}\right\}
$$

Thus as expected, in the $\tau \gg 1$ limit, the finite temperature contribution dominates.

It is straightforward to see that in this limit the system-bath dimensionless coupling is $g^{2} / T_{\sigma}^{4}$, and that the terms that we neglected in a perturbative expansion are of the order $\mathcal{O}\left(\lambda^{2}, \lambda^{2}\left(g^{2} / T_{\sigma}^{4}\right), \cdots\right)$. Recalling that the critical temperature for the scalar field $\Phi$ is given by $T_{c}^{2}=24 \mu_{R}^{2} / \lambda_{R}$, it is convenient to rescale the field and time to cast the equation in terms of dimensionless variables. Define

$$
s=\mu_{R} t \quad ; \quad \chi^{2}=\frac{\lambda_{R} \phi^{2}}{6 \mu_{R}^{2}}
$$

the equation of motion (16) becomes

$$
\begin{aligned}
& \frac{d^{2} \chi(s)}{d s^{2}}-\chi(s)\left[1-0.29 s^{3}\left(\frac{T_{\sigma}}{T_{c}}\right)^{2}\left(\frac{g^{2}}{T_{\sigma} \mu_{R}^{3}}\right)-0.31 s^{2}\left(\frac{T_{\sigma}}{T_{c}}\right)^{2}\left(\frac{g^{2}}{T_{\sigma}^{2} \mu_{R}^{2}}\right)\right] \\
& +\chi^{3}(s)-\frac{g^{2}}{m_{\sigma} \mu_{R}^{3}} \int_{0}^{s} d s^{\prime} \sin \left[\frac{m_{\sigma}}{\mu_{R}}\left(s-s^{\prime}\right)\right] \chi\left(s^{\prime}\right)=0
\end{aligned}
$$

Admittedly inserting the result from the one-loop term in the equation of motion is not quite consistent at early times because the one-loop results (26,27) were obtained for large $\tau=s\left(T_{\sigma} / \mu_{R}\right)$. However, by integrating the above equation we will obtain a qualitative understanding of the time evolution of the order parameter.

Roughly speaking, the phase transition from the broken symmetry state to the disordered phase takes place when the effective time dependent mass (18) becomes zero. This would certainly be the case in the absence of the non-Markovian term (retarded kernel in (16)). The effect of this term may be understood by expanding the field around the broken symmetry values $\phi_{ \pm}= \pm \sqrt{6 \mu_{R}^{2} / \lambda_{R}}$, and considering the deviation from these values as perturbations. One finds that the retarded kernel plays the role of a forcing term in the equation of motion for the deviation, that tends to shift the value of the minima further away from the origin.

However, we still expect that the phase transition will occur at time scales when the effective mass term vanishes, as the oscillations of the field will now occur near zero. 
From the above effective equation of motion we find that the effective mass (18) vanishes at the "critical time" $t_{c}$ given by

$$
t_{c} \approx \frac{1}{T_{\sigma}}\left[3.5\left(\frac{T_{\sigma}^{4}}{g^{2}}\right) \frac{T_{c}^{2}}{T_{\sigma}^{2}}\right]^{\frac{1}{3}}
$$

This is one of the main results of this work. Although the time scale is determined by the temperature of the heat bath, the critical time is a non-analytic function of the coupling between the system and bath.

Figure(3) shows the evolution of the equation of motion (29) for the (arbitrary) value of the parameters $\mu_{R}=m_{\sigma} ; g=m_{\sigma}^{2} ; T_{\sigma}=T_{c}=10 m_{\sigma}$. For these values, the critical time predicted by (30) is $\mu_{R} t_{c} \approx 5.8$, quite consistent with the time at which the field crosses zero in Figure (3).

Thus we see that for $T_{\sigma} \approx T_{c}$ and $g^{2} / T_{\sigma}^{4} \ll 1, t_{c} \gg 1 / T_{\sigma}$ (or alternatively $\tau_{c} \gg 1$ ) quite consistent with our original assumptions.

Notice that the critical time is non-perturbative in terms of the system-bath coupling, in a sense very much like the critical temperature is non-perturbative in terms of the scalar field self-coupling. One would then argue that the higher order corrections will only contribute perturbatively to the critical time. This argument however ignores the possible infrared divergences arising near the phase transition as it happens in equilibrium finite temperature field theory near the critical point. So we expect that, very much as it happens in equilibrium finite temperature, approaching the critical point or "critical time" in a reliable manner will require a non-perturbative resummation of the infrared sensitive diagrams [30, 31, 32]. Thus just as the critical temperature signals the breakdown of perturbation theory to study the phase transition, this critical time may also signal the breakdown of perturbation theory in the system-bath coupling. Presumably a non-perturbative resummation technique would have to be invoked to study reliably the system-bath dynamics for times close to the critical time $t_{c}$.

This possibility would have to be studied further and is beyond the scope of this article.

\section{Semiclassical Langevin Equation}

A phenomenological but rather successful approach to study the non-equilibrium dynamics of a particle coupled to a heat bath in classical statistical mechanics is provided by the Langevin equation. This approach provides a satisfactory description of Brownian motion of a particle. The Langevin equation is the classical equation of motion modified phenomenologically by (basically two) terms that account for the interaction with the heat bath. A term 
proportional to the velocity of the particle that incorporates "friction" and dissipation, and a stochastic force term that reflects the random "kicks" of the heat bath upon the Brownian particle. In most applications, this stochastic noise is assumed to be "white", that is completelety uncorrelated. The coefficient in the friction term determines the relaxation time of the Brownian particle. The friction term and the stochastic force are ultimately responsible for the approach to equilibrium of the particle with the heat bath, and are thus related by the fluctuation-dissipation theorem.

A very clear microscopic description leading to Langevin dynamics within the context of one-particle quantum mechanics has been presented by Caldeira and Leggett and Schmid 16, 33. Their study reveals that in general the friction term arises as a local approximation to a non-Markovian kernel for a particular choice for the density of states of the heat-bath, and that the noise term is typically correlated over time scales determined by the typical scales of the bath.

An attempt to obtain a microscopic description of Langevin dynamics in field theory has been reported by Morikawa[34] who considered a "fermionic bath".

In this section we offer a semiclassical derivation of the Langevin equation for the case of a bosonic heat-bath, emphasizing its range of validity, and the formal steps to go beyond the semiclassical Langevin equation.

Phenomenological Langevin equations have been recently used to study the dynamics of semiclassical configurations in a presence of a heat bath 13, 14, 15. Typically these Langevin descriptions assume a friction term and a "white-noise". We will show in what follows, that these assumptions may not be justified in many cases, and that a physically correct Langevin description must necessarily incorporate details of the particular "heat-bath".

We now consider the case in which the $\Phi$ field is initially at zero temperature, and it is coupled at $t=0$ to the heat bath, again modelled by the free field $\sigma$. The initial density matrix is now

$$
\hat{\rho}(0)=\left(|0\rangle_{\Phi}\left\langle\left. 0\right|_{\Phi}\right) \otimes e^{-\beta_{\sigma} H_{\sigma}}\right.
$$

Evolving this density matrix in time as in the previous section (details are provided in the appendices), and tracing over the bath variables (thus obtaining the influence functional) we obtain the following generating functional

$$
Z[0]=\int D \Phi_{1} \int \mathcal{D} \Phi^{+} \mathcal{D} \Phi^{-}\left\{e^{i \int_{-\infty}^{\infty} d^{4} x \mathcal{L}_{\Phi}\left[\Phi^{+}\right]-\mathcal{L}_{\Phi}\left[\Phi^{-}\right]}\right\} \times \mathcal{F}\left[\Phi^{+}, \Phi^{-}\right]
$$

with the boundary conditions $\Phi^{+}(\vec{r}, t=\infty)=\Phi^{-}(\vec{r}, t=\infty)=\Phi_{1}(\vec{r})$. 
At this stage it proves convenient to introduce the "center-of-mass" and "relative" field coordinates $\Psi$ and $\eta, \Psi=\frac{1}{2}\left[\Phi^{+}+\Phi^{-}\right] ; \eta=\left[\Phi^{+}-\Phi^{-}\right]$. These are recognized as the coordinates used in the Wigner transform of a density matrix [18]. The boundary conditions on the fields now become $\Psi(\vec{r}, t=\infty)=\Phi_{1} ; \eta(\vec{r}, t=\infty)=0$, the integral over $\Phi_{1}$, just defines the path integral over all field configurations, and finally the partition function (32) becomes

$$
\begin{aligned}
Z[0]= & \int \mathcal{D} \Psi \mathcal{D} \eta e^{i S[\Psi, \eta]} \\
S[\Psi, \eta]= & \int_{-\infty}^{\infty} d^{4} x_{1} \eta\left(x_{1}\right)\left[\left(-\square-\left(-\mu^{2}\right)\right) \Psi\left(x_{1}\right)-\frac{\lambda}{3 !} \Psi^{3}\left(x_{1}\right)\right. \\
& \left.-2 g^{2} \int_{-\infty}^{\infty} d^{4} x_{2} \Theta\left(t_{1}-t_{2}\right) \mathcal{K}_{I}\left(\vec{r}_{1}, t_{1} ; \vec{r}_{2}, t_{2}\right) \Psi\left(\vec{r}_{2}, t_{2}\right)\right]-\frac{\lambda}{4 !} \int_{-\infty}^{\infty} d^{4} x_{1} \Psi\left(x_{1}\right) \eta^{3}\left(x_{1}\right)+ \\
& i \frac{g^{2}}{2} \int_{-\infty}^{\infty} d^{4} x_{1} \int_{-\infty}^{\infty} d^{4} x_{2} \eta\left(x_{1}\right) \mathcal{K}_{R}\left(\vec{r}_{1}, t_{1} ; \vec{r}_{2}, t_{2}\right) \eta\left(x_{2}\right)
\end{aligned}
$$

where the kernels $\mathcal{K}_{R}, \mathcal{K}_{I}$ are spatially translationally invariant and their spatial Fourier transforms are given by (see appendix A).

$$
\begin{aligned}
\mathcal{K}_{R}\left(\vec{k}, t_{1}, t_{2}\right) & =\frac{1}{2 \tilde{\omega}_{k}} \cos \left[\tilde{\omega}_{k}\left(t_{1}-t_{2}\right)\right]\left[1+2 n_{\sigma}\left(\tilde{\omega}_{k}\right)\right] \Theta\left(t_{1}\right) \Theta\left(t_{2}\right) \\
\mathcal{K}_{I}\left(\vec{k}, t_{1}, t_{2}\right) & =-\frac{1}{2 \tilde{\omega}_{k}} \sin \left[\tilde{\omega}_{k}\left(t_{1}-t_{2}\right)\right] \Theta\left(t_{1}\right) \Theta\left(t_{2}\right)
\end{aligned}
$$

with $\tilde{\omega}_{k}$ being the frequencies of the heat-bath. These kernels arise from the two-point correlation function of the heat-bath fields, the real $\mathcal{K}_{R}$ and imaginary part $\mathcal{K}_{I}$ are related by the fluctuation-dissipation theorem.

To make contact with Langevin dynamics, it becomes convenient to cast the quadratic term for the $\eta$ field as resulting from a Gaussian integral over a stochastic noise term with probability distribution given by

$$
\mathcal{P}[\xi]=\exp \left[-\frac{1}{2 g^{2}}\left\{\int_{-\infty}^{\infty} d^{4} x_{1} \int_{-\infty}^{\infty} d^{4} x_{2} \xi\left(x_{1}\right) \mathcal{K}_{R}^{-1}\left(x_{1}, x_{2}\right) \xi\left(x_{2}\right)\right\}\right]
$$

Now the final form for the partition function becomes

$$
Z[0]=\int \mathcal{D} \xi \int \mathcal{D} \Psi \mathcal{D} \eta \mathcal{P}[\xi] e^{i S_{e f f}[\Psi, \eta, \xi]}
$$

with the effective action $S_{\text {eff }}$ given by

$$
S_{e f f}[\Psi, \eta, \xi]=\int d^{4} x_{1} \eta\left(x_{1}\right)\left[\left(-\square-\left(-\mu^{2}\right)\right) \Psi\left(x_{1}\right)-\frac{\lambda}{3 !} \Psi^{3}\left(x_{1}\right)\right.
$$




$$
\begin{aligned}
& \left.-2 g^{2} \int d^{4} x_{2} \Theta\left(t_{1}-t_{2}\right) \mathcal{K}_{I}\left(\vec{r}_{1}, t_{1} ; \vec{r}_{2}, t_{2}\right) \Psi\left(x_{2}\right)+\xi\left(x_{1}\right)\right] \\
& -\frac{\lambda}{4 !} \int d^{4} x \Psi(x) \eta^{3}(x)
\end{aligned}
$$

A semiclassical approximation to the above partition function requires the configurations that extremize the effective action. In particular the condition $\delta S_{\text {eff }} / \delta \eta=0$ leads to the lowest order semiclassical result

$$
\begin{aligned}
\left(\square+\left(-\mu^{2}\right)\right) \Psi\left(\vec{r}_{1}, t_{1}\right)+ & \frac{\lambda}{3 !} \Psi^{3}\left(\vec{r}_{1}, t_{1}\right)+ \\
& 2 g^{2} \int d t_{2} d^{3} r_{2} \Theta\left(t_{1}-t_{2}\right) \mathcal{K}_{I}\left(\vec{r}_{1}, t_{1} ; \vec{r}_{2}, t_{2}\right) \Psi\left(\vec{r}_{2}, t_{2}\right)=\xi\left(\vec{r}_{1}, t_{1}\right)
\end{aligned}
$$

with the Gaussian noise correlation function

$$
\left\langle\xi\left(\vec{r}_{1}, t_{1}\right) \xi\left(\vec{r}_{2}, t_{2}\right)\right\rangle=g^{2} \mathcal{K}_{R}\left(\vec{r}_{1}, t_{1} ; \vec{r}_{2}, t_{2}\right)
$$

This is the typical Langevin equation. This equation, however, is not the only dynamical evolution equation in the semiclassical limit, other equations are obtained by performing the variational derivatives with respect to the "noise" term and $\Psi$. Langevin dynamics results from the decoupling approximation to these equations, in particular neglecting the coupling between $\Psi$ and $\eta$ and $\eta$ and the "noise". A consistent improvement over the semiclassical Langevin equation will involve a perturbative expansion in these terms. Allowing for the couplings of the different fields to the "noise" term will introduce corrections to the "noise" correlation functions. This corrections are a manifestation of the "back-reaction" of the system on the bath correlations.

One may obtain an equation of motion similar to the effective equation found in the previous section for the order parameter (16) from the above Langevin equation by splitting the field as

$$
\Psi(\vec{r}, t)=\phi(t)+\varphi(\vec{r}, t)
$$

requiring that the "mean field" $\phi(t)$ obeys a source free equation and considering $\varphi$ as the fluctuation whose equation of motion contains the noise term. The linearized equation for the fluctuation with the noise term, and non-Markovian kernel may be solved by introducing the retarded propagator. In the equation for the "mean-field" there is a term of the form

$$
\frac{\lambda}{2} \phi(t)\left(\varphi\left(\vec{r}_{1}, t\right)\right)^{2}
$$


By taking the average of this term over the noise with the noise auto-correlation function (39) one obtains the $\mathcal{O}\left(\lambda g^{2}\right)$ correction to the equation of motion of the order parameter, but not the one-loop correction without the bath $(\mathcal{O}(\lambda))$. This semiclassical Langevin equation does not incorporate quantum loop corrections of the scalar self-interaction.

There are two features of the semiclassical Langevin equation that deserve comment: the first is that the non-Markovian kernel does not lead to dissipation. Secondly, the noise does not have "white" (delta functions) correlations. In fact these two features are related by the fluctuation-dissipation theorem.

The fact that there is no dissipation is a consequence of the simplicity of the model, one could try a coupling to the heat-bath that is linear in the scalar field and quadratic in terms of the bath fields. Integration over the bath fields will also yield to a non-local influence functional which however will not be quadratic in the scalar field, but may be studied perturbatively. The quadratic contribution of the scalar field will involve a "bubble" diagram from the bath fields. This diagram will have a two-particle threshold that may lead to dissipative processes, which however, is very high in energy if the bath particles are much heavier than the system particles. Thus in this case long wavelength low frequency components of the field will evolve without dissipation.

Such a diagram appears naturally in the case in which the bath field corresponds to fermions coupled to the scalar field via a Yukawa coupling. A memoriless (Markovian) friction term in the Langevin equation (proportional to the time derivative of the field) may be obtained at low frequencies provided the non-local kernel has a power series expansion in the transferred frequency with a linear term in the frequency. The coefficient of this linear term will be the friction coefficient. From the above discussion, it is clear that this possibility is not very easy to achieve if the bath particles are more massive than the system particles. In this case the multiparticle threshold will be very high in frequency and there will be no dissipation for low transferred frequency. This may be inferred from the work of Morikawa 34]. This situation may change if there are collective excitations in the bath, that produce a linear frequency dependence of the non-local kernel at low frequencies. This scenario, however, is not generic and will depend on the details of the heat bath.

This conclusion is particularly meaningful within the context of Langevin dynamics of semiclassical field configurations. These are large amplitude coherent configurations, but typically slowly varying in space and time, thus corresponding to small frequency and momentum transfers to the bath. Although very massive, because they are mainly composed of long-wavelength, low frequency modes, it is difficult for these configurations to "decay" and thus dissipate. 
Derivative couplings between system and bath fields may lead to dissipative terms, but are very dangerous from the point of view of renormalizability. Couplings that are quadratic (or higher order polynomials) in the scalar field will yield to higher order polynomials in the influence functional and certainly will not lead to a simple Langevin description.

Thus in order to obtain a semiclassical Langevin description there is a strong restriction on the system-bath coupling: the system must couple linearly to the bath, so that the influence functional is quadratic. A friction term may arise if the bath degrees of freedom are lighter than the system's degrees of freedom, but as discussed by Morikawa 34 this will have to be studied case by case, in particular it may require a higher order calculation.

The point of this discussion is that writing down a simple Langevin equation with a local dissipative term and a noise term with white correlations may have to be justified by looking at the particular models in detail.

\section{Conclusions:}

We have studied the dynamics of a phase transition in which a scalar field theory (the system), initially in the ordered (broken symmetry) phase at low temperatures is coupled to a heat bath. This heat-bath is represented by another scalar field, linearly coupled to the "system field" and whose temperature is close to the critical temperature for the system. We derived, the one-loop effective equations of motion for the order parameter that incorporates the effects of the heat bath.

The heat bath introduces a non-Markovian, non-dissipative kernel that is temperature independent and also introduces one-loop corrections that are temperature dependent.

The effective evolution equations were integrated and an estimate of the time to complete the phase transition to the symmetric phase was obtained. This time is a non-analytic function of the "system-bath" coupling and may signal the breakdown of perturbation theory in terms of this coupling.

We derived a semiclassical Langevin equation for the system, it contains a non-Markovian, non-dissipative kernel, and the noise term, although Gaussian (in this approximation), is colored and correlated on time scales of the order of the inverse temperature of the heat-bath, when this temperature is much larger than the masses of the fields. It is pointed out that the non-Markovian kernel will be model dependent and may only be approximated by a local dissipative (friction) term only in very special cases. The derivation of the Langevin equation permits to identify a formal expansion to improve over the semiclassical approximation and to account for quantum effects (loops) of the system field, as well as for back reaction of the 
system on the bath.

Acknowledgments: The authors would like to thank D. Jasnow, R. Willey, A. Bochkarev, R. Carlitz, R. Holman, A. Weldon and J. Kapusta for very enlightening conversations and comments. They also thank the N.S.F for partial support through Grant No: PHY-8921311. D.-S. Lee acknowledges partial support through a Mellon PreDoctoral Fellowship Award. 


\section{Appendix A : Finite Temperature Generating Functionals}

In this appendix, we summarize the most relevant technical details leading to the generating functional (12) in section 2. Most of the steps may be found in the literature [25, 26, 27, 28, 29, however we present here the generalizations and modifications appropriate to our case in order to make our article self-contained.

Starting from the generating functional (10) let us insert the resolution of the identity in terms of a complete set of field eigenstates

$$
\int D \Phi D \sigma|\Phi, \sigma\rangle\langle\sigma, \Phi|=1
$$

between all the time evolution operators, obtaining

$$
\begin{aligned}
Z\left[J_{\Phi}^{+}, J_{\Phi}^{-}, J_{\Phi}^{\beta}\right]= & \int D \Phi_{1} D \Phi_{2} D \Phi_{3} \int D \sigma_{1} D \sigma_{2} D \sigma_{3} \\
& \left\{\left\langle\Phi_{1}, \sigma_{1}\left|U_{\Phi}\left(T-i \beta_{\Phi}, T ; J_{\Phi}^{\beta}\right) U_{\sigma}\left(T-i \beta_{\sigma}, T\right)\right| \Phi_{2}, \sigma_{2}\right\rangle\right\} \times \\
& \left\{\left\langle\Phi_{2}, \sigma_{2}\left|U\left(T, T^{\prime} ; J_{\Phi}^{-}\right)\right| \Phi_{3}, \sigma_{3}\right\rangle\left\langle\Phi_{3}, \sigma_{3}\left|U\left(T^{\prime}, T ; J_{\Phi}^{+}\right)\right| \Phi_{1}, \sigma_{1}\right\rangle\right\}
\end{aligned}
$$

Each matrix element in the above expression has a functional integral representation. Then, the generating function becomes (to avoid cluttering of notation we suppress the space-time indices)

$$
\begin{aligned}
Z\left[J_{\Phi}^{+}, J_{\Phi}^{-}, J_{\Phi}^{\beta}\right]= & \int D \Phi_{1} D \Phi_{2} D \Phi_{3} \int \mathcal{D} \Phi^{+} \mathcal{D} \Phi^{-} \mathcal{D} \Phi^{\beta} \\
& \left\{e^{i \int_{T}^{T^{\prime}} \mathcal{L}_{\Phi}\left[\Phi^{+}\right]+J_{\Phi}^{+} \Phi^{+}-\mathcal{L}_{\Phi}\left[\Phi^{-}\right]-J_{\Phi}^{-} \Phi^{-}}\right\}\left\{e^{i \int_{T}^{T-i \beta_{\Phi}} \mathcal{L}_{\Phi}\left[\Phi^{\beta}\right]+J_{\Phi}^{\beta} \Phi^{\beta}}\right\} \times \mathcal{F}\left[\Phi^{+}, \Phi^{-}\right]
\end{aligned}
$$

with the boundary conditions $\Phi^{+}(T)=\Phi^{\beta}\left(T-i \beta_{\Phi}\right)=\Phi_{1} ; \Phi^{-}(T)=\Phi^{\beta}(T)=\Phi_{2}$ and $\Phi^{+}\left(T^{\prime}\right)=\Phi^{-}\left(T^{\prime}\right)=\Phi_{3}$. This can be regarded as a path integral along the contour $(C)$ in the complex time plane shown in Figure (1) with periodic boundary conditions.

The non-local functional $\mathcal{F}$ is the "influence functional" [18, [16] obtained by tracing-out the bath degrees of freedom $(\sigma)$

$$
\begin{aligned}
\mathcal{F}\left[\Phi^{+}, \Phi^{-}\right]= & \int D \sigma_{1} D \sigma_{2} D \sigma_{3} \int \mathcal{D} \sigma^{+} \mathcal{D} \sigma^{-} \mathcal{D} \sigma^{\beta} \\
& \left\{e^{i \int_{T}^{T^{\prime}} \mathcal{L}_{\sigma}\left[\sigma^{+}\right]+\mathcal{L}_{I}\left[\sigma^{+}, \Phi^{+}\right]-\mathcal{L}_{\sigma}\left[\sigma^{-}\right]-\mathcal{L}_{I}\left[\sigma^{-}, \Phi^{-}\right]}\right\}\left\{e^{i \int_{T}^{T-i \beta \Phi} \mathcal{L}_{\sigma}\left[\sigma^{\beta}\right]}\right\}
\end{aligned}
$$


with the boundary conditions $\sigma^{+}(T)=\sigma^{\beta}\left(T-i \beta_{\sigma}\right)=\Phi_{1} ; \sigma^{-}(T)=\sigma^{\beta}(T)=\sigma_{2}$ and $\sigma^{+}\left(T^{\prime}\right)=$ $\sigma^{-}\left(T^{\prime}\right)=\sigma_{3}$. The normalization term $\mathcal{F}[0,0]$ will cancel against the denominator in the computation of correlation functions. The influence functional can also be recognized as a path integral along the contour $(C)$ shown in Figure (1).

For the linear coupling between system and bath fields, the trace (path integral) over the bath variables can be done at once leading to the expression (11).

The propagators $D_{\sigma}^{a b}(a, b=+,-)$ are constructed from the functions $D_{\sigma}^{>}, D_{\sigma}^{<}$which are the homogeneous solutions of the quadratic form in $\mathcal{L}_{\sigma}$ obeying the Kubo-Martin-Schwinger boundary condition (periodicity in imaginary time)

$$
D_{\sigma}^{>}\left(\vec{r}_{1}, t_{1}-i \beta_{\sigma} ; \vec{r}_{2}, t_{2}\right)=D_{\sigma}^{<}\left(\vec{r}_{1}, t_{1} ; \vec{r}_{2}, t_{2}\right)
$$

The spatial Fourier transforms of these functions are given by

$$
\begin{aligned}
i D_{\sigma}^{>}\left(\vec{k} ; t, t^{\prime}\right) & =\frac{1}{2 \tilde{\omega}_{k}}\left\{\left(1-n_{\sigma}\left(\tilde{\omega}_{k}\right)\right) e^{-i \tilde{\omega}_{k}\left(t-t^{\prime}\right)}+n_{\sigma}\left(\tilde{\omega}_{k}\right) e^{i \tilde{\omega}_{k}\left(t-t^{\prime}\right)}\right\} \\
D_{\sigma}^{>}\left(\vec{k} ; t, t^{\prime}\right) & =D_{\sigma}^{<}\left(\vec{k}, t^{\prime}, t\right) \\
n_{\sigma}\left(\tilde{\omega}_{k}\right) & =\frac{1}{e^{\beta_{\sigma} \tilde{\omega}_{k}}-1} ; \quad \tilde{\omega}_{k}=\sqrt{\vec{k}^{2}+m_{\sigma}^{2}}
\end{aligned}
$$

The Green's functions $\left(D_{\sigma}^{a b}\left(t_{1}, t_{2}\right)\right)$ that enter in the integral are now explicitly given by

$$
\begin{aligned}
& D_{\sigma}^{++}\left(\vec{r}_{1}, t_{1} ; \vec{r}_{2}, t_{2}\right)=D_{\sigma}^{>}\left(\vec{r}_{1}, t_{1} ; \vec{r}_{2}, t_{2}\right) \Theta\left(t_{1}-t_{2}\right)+D_{\sigma}^{<}\left(\vec{r}_{1}, t_{1} ; \vec{r}_{2}, t_{2}\right) \Theta\left(t_{2}-t_{1}\right) \\
& D_{\sigma}^{--}\left(\vec{r}_{1}, t_{1} ; \vec{r}_{2}, t_{2}\right)=D_{\sigma}^{>}\left(\vec{r}_{1}, t_{1} ; \vec{r}_{2}, t_{2}\right) \Theta\left(t_{2}-t_{1}\right)+D_{\sigma}^{<}\left(\vec{r}_{1}, t_{1} ; \vec{r}_{2}, t_{2}\right) \Theta\left(t_{1}-t_{2}\right) \\
& D_{\sigma}^{+-}\left(\vec{r}_{1}, t_{1} ; \vec{r}_{2}, t_{2}\right)=-D_{\sigma}^{<}\left(\vec{r}_{1}, t_{1} ; \vec{r}_{2}, t_{2}\right) \\
& D_{\sigma}^{-+}\left(\vec{r}_{1}, t_{1} ; \vec{r}_{2}, t_{2}\right)=-D_{\sigma}^{>}\left(\vec{r}_{1}, t_{1} ; \vec{r}_{2}, t_{2}\right)=-D_{\sigma}^{<}\left(\vec{r}_{1}, t_{1} ; \vec{r}_{2}, t_{2}\right)
\end{aligned}
$$

As usual, the path integral over the quadratic form may be evaluated and we may then expand all interaction terms perturbatively for weak couplings.

After some straightforward algebra we obtain the following partition function

$$
\begin{aligned}
Z\left[J_{\Phi}^{+}, J_{\Phi}^{-}, J_{\Phi}^{\beta}\right]= & \exp \left\{i \frac{\lambda}{4 !} \int_{T}^{T^{\prime}} d t\left[\left(-i \frac{\delta}{\delta J_{\Phi}^{+}}\right)^{4}-\left(i \frac{\delta}{\delta J_{\Phi}^{-}}\right)^{4}\right]\right\} \exp \left\{i \frac{\lambda}{4 !} \int_{T}^{T-i \beta_{\Phi}} d t\left(-i \frac{\delta}{\delta J_{\Phi}^{\beta}}\right)^{4}\right\} \\
& \mathcal{F}\left[-i \frac{\delta}{\delta J_{\Phi}^{+}}, i \frac{\delta}{\delta J_{\Phi}^{-}}\right] \times \exp \left\{-\frac{i}{2} \int_{C} d t_{1} \int_{C} d t_{2} J_{\Phi}^{C}\left(t_{1}\right) D_{\Phi}^{C}\left(t_{1}, t_{2}\right) J_{\Phi}^{C}\left(t_{2}\right)\right\}
\end{aligned}
$$

where $J_{\Phi}^{C}$ stands for the source term on the contour $C$. 
In the limit $T^{\prime} \rightarrow \infty$ and, $T \rightarrow-\infty$ the contributions from the terms in which one of the currents is $J_{\Phi}^{+}$or $J_{\Phi}^{-}$and the other is a $J_{\Phi}^{\beta}$ vanish when computing correlation functions in which the external legs are at finite real time, as a consequence of the Riemann-Lebesgue lemma 20]. For this real time correlation functions, there is no contribution from the $J_{\Phi}^{\beta}$ terms that cancel between numerator and denominator. Then the calculation of finite real time correlation functions, the generating functional simplifies to

$$
\begin{aligned}
Z\left[J_{\Phi}^{+}, J_{\Phi}^{-}\right]= & \exp \left\{i \frac{\lambda}{4 !} \int_{-\infty}^{\infty} d t\left[\left(-i \frac{\delta}{\delta J_{\Phi}^{+}}\right)^{4}-\left(i \frac{\delta}{\delta J_{\Phi}^{-}}\right)^{4}\right]\right\} \mathcal{F}\left[-i \frac{\delta}{\delta J_{\Phi}^{+}}, i \frac{\delta}{\delta J_{\Phi}^{-}}\right] \times \\
& \exp \left\{-\frac{i}{2} \int_{-\infty}^{\infty} d t_{1} \int_{-\infty}^{\infty} d t_{2} J_{\Phi}^{a}\left(t_{1}\right) D_{\Phi}^{a b}\left(t_{1}, t_{2}\right) J_{\Phi}^{a}\left(t_{2}\right)\right\}
\end{aligned}
$$

with $a, b=+,-$.

The Green's functions $D_{\Phi}^{a b}$ are similar to (46,47) and obtained from them by replacing $m_{\sigma} ; \beta_{\sigma}$ by the values for the $\Phi$ field.

\section{Appendix B: Evolution equations}

The most convenient method to obtain the evolution equations is the tadpole method [30]. In the present non-equilibrium situation, it is implemented in the following manner. First we recall that the non-equilibrium generating function without sources is given by

$$
Z[0]=\int D \Phi_{1} D \Phi_{2} D \Phi_{3} \int \mathcal{D} \Phi^{+} \mathcal{D} \Phi^{-} \mathcal{D} \Phi^{\beta} \quad e^{i \mathcal{S}_{e f f}\left[\Phi^{+}, \Phi^{-}, \Phi^{\beta}\right]}
$$

with the boundary conditions $\Phi^{+}(T)=\Phi^{\beta}\left(T-i \beta_{\Phi}\right)=\Phi_{1} ; \Phi^{-}(T)=\Phi^{\beta}(T)=\Phi_{2}$ and $\Phi^{+}\left(T^{\prime}\right)=\Phi^{-}\left(T^{\prime}\right)=\Phi_{3}$. After integrating out the degrees of freedom of field $\sigma$ (bath), $\mathcal{S}_{\text {eff }}$ including the influence functional is thus

$$
\begin{aligned}
\mathcal{S}_{\text {eff }}\left[\Phi^{+}, \Phi^{-}, \Phi^{\beta}\right] & =\int_{T}^{T^{\prime}} d t\left\{\mathcal{L}_{\Phi}\left[\Phi^{+}\right]-\mathcal{L}_{\Phi}\left[\Phi^{-}\right]\right\}+\int_{T}^{T-i \beta_{\Phi}} d t \mathcal{L}_{\Phi}\left[\Phi^{\beta}\right] \\
& -\frac{1}{2} g^{2} \int_{0}^{T^{\prime}} d t_{1} \int_{0}^{T^{\prime}} d t_{2} \Phi^{a}\left(t_{1}\right) D_{\sigma}^{a b}\left(t_{1}, t_{2}\right) \Phi^{a}\left(t_{2}\right)
\end{aligned}
$$

We shift the $\Phi^{ \pm}$fields by

$$
\Phi^{ \pm}(\vec{r}, t)=\phi(t)+\hat{\Phi}^{ \pm}(\vec{r}, t)
$$

where $\phi$ is a background mean field. The tadpole method [30] requires that

$$
\left\langle\hat{\Phi}^{ \pm}(\vec{r}, t)\right\rangle=0
$$

We also need to shift the field $\Phi^{\beta}$ by

$$
\Phi^{\beta}(\vec{r}, t)=\phi(T)+\hat{\Phi}^{\beta}(\vec{r}, t)
$$


Prior to the time when the system is coupled to the bath, the system is in equilibrium and $\phi$ is a constant in time. So for $t \leq 0, \phi(t)=\phi(0)$ which is the initial equilibrium value of the mean field.

Now we expand

$$
\mathcal{L}(\Phi)=\mathcal{L}(\phi)+\frac{\delta \mathcal{L}}{\delta \Phi} \Phi+\frac{1}{2} \frac{\delta^{2} \mathcal{L}}{\delta \Phi^{2}} \Phi^{2}+\frac{1}{3 !} \frac{\delta^{3} \mathcal{L}}{\delta \Phi^{3}} \Phi^{3}+\cdots
$$

for the $(+,-, \beta)$ branches and consider the linear, cubic and quartic terms in $\Phi$ as perturbations. The sources are now coupled to the fluctuation part of the field to generate the perturbative expansion and require that order by order in perturbation theory the tadpole condition (53) is fulfilled.

An important ingredient is the identity

$$
D^{++}+D^{+-}+D^{-+}+D^{--}=0
$$

for both the $\sigma$ and $\Phi$ Green's functions.

\section{References}

[1] E. W. Kolb and M. S. Turner, "The Early Universe", Addison Wesley (Frontiers in Physics) (1990)

[2] See for example A. Linde, Particle Physics and Inflationary Cosmology, Harwood Academic Publishers (1990), and references therein.

[3] R. H. Brandenberger, Rev. of Mod. Phys. 57, 1 (1985); Int. J. Mod. Phys. 2A, 77 (1987).

[4] A. A. Starobinsky, in Field Theory, Quantum Gravity and Strings, Proceedings of the Seminar Series, Meudon and Paris, France, 1984, Eds. H. J. de Vega and N. Sanchez, Lecture Notes in Physics Vol. 246 (Springer, Berlin 1986).

[5] E. Calzetta and B. L. Hu, Phys. Rev. D 35, 495 (1987); 37, 2838 (1988); J. P. Paz, ibid. 41, 1054 (1990).

[6] B. L. Hu in Quantum Mechanics in Curved Spacetime, Ed. by J. Audretsch and V. de Sabbata (Plenum, London 1990), and Proceedings of the Second International Workshop on Thermal Fields and Their Applications, Ed. by H. Ezawa et. al. (North Holland, 1991) 
[7] B. L. Hu, J. P. Paz and Y. Zhang, Phys. Rev. D 45, 2843 (1992); ibid. 47, 1576 1993; T. A. Brun, "Semiclassical equations of motion for nonlinear brownian systems", CALT68-1848 (1993) (unpublished).

[8] W. H. Zurek, in Frontiers in Nonequilibrium Statistical Physics, Ed. G. T. Moore and M. O. Scully (Plenum, N.Y. 1986); Phys. Rev. D 24, 1516 (1981); 26, 1862 (1982); W. G. Unruh and W. H. Zurek, Phys. Rev. D 40, 1071 (1989). G. W. Ford, J. T. Lewis and R.F. O'Connell, Phys. Rev. A 37, 4419 (1988).

[9] R. D. Carlitz and R. Chakrabarti, Phys. Rev. D 31, 1418, (1985).

[10] J. Cloutier and G. W. Semenoff, Phys. Rev. D 44, 3218 (1991). These authors consider symmetry restoration by coupling linearly to a noise rather than another field that plays the role of a heat bath.

[11] D. Boyanovsky and H. J. de Vega "Quantum rolling down out of equilibrium", (to appear in Phys. Rev. D)

[12] D. Boyanovsky, D.-S. Lee and A. Singh "Phase Transitions out of equilibrium: domain formation and growth" (submitted); D. Boyanovsky "Quantum spinodal decomposition" (submitted).

[13] M. Alford, H. Feldman and M. Gleiser, "Testing classical nucleation theory" (NSF-ITP1992) (unpublished).

[14] A. Bochkarev and P. de Forcrand, "Non-perturbative evaluation of the diffusion rate in field theory at high temperatures" P. R. D. (to appear, 1993); Phys.Rev. D 44, 519 (1991); Phys. Rev. Lett. 63, 2337 (1989).

[15] M. Alford, H. Feldman, and M. Gleiser, Phys. Rev. Lett. 68, 1645 (1992).

[16] A. O. Caldeira and A. J. Leggett, Physica A 121, 587 (1983).

[17] J. P. Paz in Proceedings of the Second International Workshop on Thermal Fields and Their Applications. Ed. H. Ezawa et. al. (North Holland, 1991).

[18] R. P. Feynman and F. L. Vernon, Jr. Ann. Phys. (N.Y.) 24, 118 (1963).

[19] H. Grabert, P. Schramm and G. L. Ingold, Phys. Rep. 168, 115 (1988).

[20] R. Mills, "Propagators for Many Particle Systems" (Gordon and Breach, N. Y. 1969). 
[21] J. Schwinger, J. Math. Phys. 2, 407 (1961).

[22] L. V. Keldysh, Sov. Phys. JETP 20, 1018 (1965).

[23] N. P. Landsman and C. G. van Weert, Phys. Rep. 145, 141 (1987).

[24] G. Semenoff and N. Weiss, Phys. Rev. D31, 689; 699 (1985).

[25] R. D. Jordan, Phys. Rev. D33, 444 (1986).

[26] A. Niemi and G. Semenoff, Ann. of Phys. (N.Y.) 152, 105 (1984); Nucl. Phys. B [FS10], 181, (1984).

[27] R. L. Kobes and K. L. Kowalski, Phys. Rev. D34, 513 (1986); R. L. Kobes, G. W. Semenoff and N. Weiss, Z. Phys. C 29, 371 (1985).

[28] E. Calzetta, Ann. of Phys. (N.Y.) 190, 32 (1989)

[29] E. Calzetta and B. L. Hu, Phys. Rev. D35, 495 (1987); Phys. Rev. D37, 2878 (1988).

[30] S. Weinberg, Phys. Rev. D9, 3357 (1974). (1985).

[31] L. Dolan and R. Jackiw, Phys. Rev. D 9, 3320 (1974)

[32] J. I. Kapusta, "Finite Temperature Field Theory", Cambridge Univ. Press (1989).

[33] A. Schmid Jour. of Low Temp. Phys. 49, 609 (1982).

[34] M. Morikawa, Phys. Rev. D. 33, 3607 (1986). 


\section{Figure Captions:}

Figure 1: Contour in the complex time plane for the non-equilibrium generating functional.

Figure 2: a) Propagators $(++)$ for the "system" field (straight line) and "bath" field (wavy line), there are four of each $(++;+-;-+;--)$. b) One loop correction to $\mathcal{O}\left(\lambda g^{2}\right)$. There are four terms for the insertion of the bath propagators.

Figure 3: Numerical evolution of the effective equation of motion for $\chi(s)$ vs. $s$ for the values $\mu_{R}=m_{\sigma} ; g=m_{\sigma}^{2} ; T_{\sigma}=T_{c}=10 m_{\sigma}$. The initial conditions are: $\chi(0)=1 ; \dot{\chi}(0)=0$. The solid line is the evolution of the classical field equations (without the non-Markovian kernel). The long dashed line is the evolution including the non-Markovian kernel but without the one-loop correction. The short dashed line is the full equation including the non-Markovian kernel and the one-loop contribution. 